\title{
MACDONALD CONJECTURES \\ AND THE SELBERG INTEGRAL
}

By

\author{
Laurent Habsieger
}

IMA Preprint Series \# 490

February 1989 


\title{
MACDONALD CONJECTURES AND THE SELBERG INTEGRAL
}

\author{
LAURENT HABSIEGER $\dagger$
}

Abstract. In 1962 Dyson proposed the value $(n k) ! / k !^{n}$ for the constant term in the expansion of the product $\prod_{i \neq j}\left(1-x_{i} / x_{j}\right)^{k}$. In 1980 Macdonald stated several conjectures that generalize Dyson's conjecture, by considering some products associated to root systems. We will present various forms of Macdonald's conjectures and describe the results obtained so far. A useful tool in this study is Selberg's integral, a multivariate extension of the beta integral. We will also give the connections between Selberg's integral and Macdonald's conjectures, and explain some other extensions of Selberg's integral.

\section{First Part: Dyson's conjecture.}

A. Presentation. In statistical physics Dyson [6] was led to consider the following integral:

$$
\int_{0}^{1} \ldots \int_{0}^{1} \prod_{1 \leq j<k \leq n}\left|e^{2 i \pi \theta_{j}}-e^{2 i \pi \theta_{k}}\right|^{2 z} d \theta_{1} \ldots d \theta_{n}
$$

for which he conjectured the value $\Gamma(n z+1) / \Gamma(z+1)^{n}$. Noticing that $\left|e^{2 i \pi \theta_{j}}-e^{2 i \pi \theta_{k}}\right|^{2}=$ $\left(1-e^{2 i \pi\left(\theta_{j}-\theta_{k}\right)}\right)\left(1-e^{2 i \pi\left(\theta_{k}-\theta_{j}\right)}\right)$, this is equivalent to

$$
\int_{0}^{1} \ldots \int_{0}^{1} f\left(e^{2 i \pi \theta_{1}}, \ldots, e^{2 i \pi \theta_{n}}\right) d \theta_{n} \ldots d \theta_{n}=\frac{\Gamma(n k+1)}{\Gamma(k+1)^{n}}
$$

where $f\left(x_{1}, \ldots, x_{n}\right)=\prod_{1 \leq i \neq j \leq n}\left(1-\frac{x_{i}}{x_{j}}\right)^{k}$.

So his conjecture may be stated as:

$$
C T \prod_{1 \leq \neq j \leq n}\left(1-\frac{X_{i}}{X_{j}}\right)^{k}=\frac{(n k) !}{k !^{n}}
$$

where $C T f\left(x_{1}, \ldots, x_{n}\right)$ denotes the coefficient of $x_{1}^{o} x_{2}^{o} \ldots x_{n}^{o}$ in the expansion of $f\left(x_{1}, \ldots, x_{n}\right)$. More generally he conjectured that

$$
C T \prod_{1 \leq i \neq j \leq n}\left(1-\frac{x_{i}}{x_{j}}\right)^{a_{i}}=\frac{\left(a_{1}+\cdots+a_{n}\right) !}{a_{1} ! \ldots a_{n} !}
$$

This was proved by Gunson (1962, [10]), Wilson (1962, [21]), Good (1970, [9]), Zeilberger (1982, [22]).

†This work has been done on a postdoctoral position of the Institute for Mathematics and its Applications, University of Minnesota, 514 Vincent Hall, 206 Church St. S.E., Minneapolis, MN 55455, during
the academic year 1987-88. 


\section{B. The $q$-analogue}

$$
\begin{aligned}
& \text { Let us put } \quad(x)_{\infty}=(x ; q)_{\infty}=\prod_{i=0}^{\infty}\left(1-x q^{i}\right) \\
& \text { and } \quad(x)_{n}=(x ; q)_{n}=\prod_{i=0}^{n-1}\left(1-x q^{i}\right)=\frac{(x)_{\infty}}{\left(x q^{n}\right)_{\infty}}
\end{aligned}
$$

In 1975 Andrews [1] proposed the following $q$-analogue of Dyson's conjecture :

$$
C T . \prod_{1 \leq i<j \leq n}\left(\frac{x_{i}}{x_{j}}\right)_{a_{i}}\left(q \frac{x_{j}}{x_{i}}\right)_{a_{j}}=\frac{(q)_{a_{1}}+\ldots+a_{n}}{(q)_{a_{1}} \ldots(q)_{a_{n}}}
$$

Andrews was able to prove the cases $n=2,3$ and Kadell (1983, [14]) the case $n=4$. The general case was proved by Zeilberger and Bressoud (1984, [26]), and Bressoud and Goulden (1985, [5]).

Remarks. 1) As usual, when $q$ tends to 1 , one gets the ordinary Dyson conjecture.

2) Bressoud and Goulden's proof is just an improvement of Zeilberger and Bressoud's proof, which itself extends Zeilberger's proof of Dyson's conjecture.

The main idea that occurs in Bressoud and Goulden's proof is the notion of tournament.

DEfINITION. A tournament $T$ on $n$ vertices is a set of ordered pairs $(i, j)$ such that $1 \leq i \neq j \leq n$, and $(i, j) \in T$ if and only if $(j, i) \notin T$. Moreover if the condition $[(i, j) \in T$ and $(j, k) \in T]$ implies $(i, k) \in T$, for all $1 \leq i, j, k \leq n$, the tournament is transitive. Otherwise $T$ is non-transitive.

Equivalently a transitive tournament may be viewed as $\{(\sigma(i), \sigma(j)): 1 \leq i<j \leq n\}$, for some permutation $\sigma$ of $\{1, \ldots, n\}$.

Bressoud and Goulden's "master theorem" states that

$$
C T \prod_{(i, j) \in T}\left(\frac{x_{i}}{x_{j}}\right)_{a_{i}}\left(q \frac{x_{j}}{x_{i}}\right)_{a_{j}-1}=0
$$

if $T$ is a non-transitive tournament.

When $T$ is a transitive tournament we may suppose, up to permutation, that $\sigma=i d$ and then we have

$$
C T \prod_{1 \leq i<j \leq n}\left(\frac{x_{i}}{x_{j}}\right)_{a_{i}}\left(q \frac{x_{j}}{x_{i}}\right)_{a_{j}-1}=\frac{(q)_{a_{1}}+\ldots a_{n}}{(q)_{a_{1}} \ldots(q)_{a_{n}}} \times \prod_{i=1}^{n} \frac{1-q^{a_{i}}}{1-q^{a_{1}+\ldots+a_{i}}}
$$

This has also been proved by Bressoud and Goulden but was already conjectured by Kadell in his paper about the case $n=4$. 
C. Connection with the root system $A_{n-1}$. For $1 \leq i \leq n$ let us denote by $e_{i}$ the i-th vector in the canonical basis of $R^{n}$ and let us put

$$
A_{n-1}=\left\{e_{i}-e_{j}: 1 \leq i \neq j \leq n\right\}
$$

We have $\operatorname{dim} A_{n-1}=n-1$, which explains the choice of the index. Let us also denote by $\Lambda$ the lattice spanned by $A_{n-1}$, i.e.

$$
\Lambda=\left\{\alpha=\sum_{i=1}^{n} \alpha_{i} e_{i}: \alpha_{i} \in \mathbf{Z}, \sum_{i=1}^{n} \alpha_{i}=0\right\} .
$$

For $\alpha \in \Lambda$, we will represent by $e^{\alpha}$ the corresponding element of $Z[\Lambda]:$ if $x_{i}=e^{\mathfrak{p}_{i}}$, then $e^{\alpha_{1} e_{1}+\cdots+\alpha_{n} e_{n}}=x_{1}^{\alpha_{1}} \ldots x_{n}^{\alpha_{n}}$.

The Dyson conjecture with equal parameters may be written as

$$
C T \prod_{\alpha \in A_{n-1}}\left(1-e^{\alpha}\right)^{k}=\frac{(n k) !}{k !^{n}}
$$

where $C T \sum_{\alpha \in \Lambda} a_{\alpha} e^{\alpha}=a_{0}$ is the coefficient of $e^{0 e_{1}+\cdots+0 e_{n}}$ is the expansion of the product.

The $q$-case may also be written as

$$
C T \prod_{\alpha \in A_{n-1}^{+}}\left(q e^{\alpha}\right)_{k}\left(e^{-\alpha}\right)_{k}=\frac{(q)_{n k}}{(q)_{k}^{n}}
$$

where $A_{n-1}^{+}=\left\{\alpha=e_{i}-e_{j} \in A_{n-1}: j-i>0\right\}$; the set $A_{n-1}$ is the set of all the roots, and $A_{n-1}^{+}$is the set of positive roots.

Those formulations suggested to Macdonald other conjectures, related to other root systems than $A_{n-1}$, that we will describe now.

\section{Second Part: Macdonald's and Morris's conjectures.}

A] Root Sytems. Let $V$ be an Euclidean vector space of dimension $n$, with the scalar product $\langle$,$\rangle . For each non-zero vector \alpha$ in $V$ we define $\alpha^{\vee}: V \rightarrow \mathbf{R}$ and $w_{\alpha}: V \rightarrow V$ by:

$$
\alpha^{\vee}(\beta)=\frac{2\langle\alpha, \beta\rangle}{\langle\alpha, \alpha\rangle} \text { and } w_{\alpha}(\beta)=\beta-\alpha^{\vee}(\beta) \alpha,
$$

for any vector $\beta$ in $V$. The linear map $w_{\alpha}$ is the reflection associated to $\alpha$.

A root system of $V$ is a subset $R$ of $V$ that satisfies the four following axioms:

(RS1) $\quad R$ spans $V$ and does not contain 0;

(RS2) If $\alpha \in R$, then $w_{\alpha}(R)=R$;

(RS3) If $\alpha, \beta \in R$, then $\alpha^{\vee}(\beta) \in \mathbf{Z}$;

(RS4) $R$ is finite. 
If for each $\alpha$ in $R$ we have $\{\beta \in R: \beta$ proportional to $\alpha\}=\{\alpha,-\alpha\}$, the root system $R$ is said to be reduced.

If $R$ is not the disjoint union of two non-empy orthogonal sets, the root system $R$ is said to be irreducible.

There is a classification for reduced irreducible root systems:

- four infinite families: $A_{n}, B_{n}, C_{n}, D_{n}$.

- the "exceptional" root systems: $E_{6}, E_{7}, E_{8}, F_{4}, G_{2}$.

A set of positive roots of $R$ is a subset $R^{+}$of $R$ such that:

i) $\forall \alpha \in R, \alpha \in R^{+} \Leftrightarrow-\alpha \notin R^{+}$,

ii) $\forall(\alpha, \beta) \in R^{+} \times R^{+}, \alpha+\beta \in R \Leftrightarrow \alpha+\beta \in R^{+}$.

The group spanned by the $w_{\alpha}(\alpha \in R)$ is denoted by $W$ and is called the Weyl group of $R$. The group $W$ acts naturally on the symmetric algebra of $V$ and the subalgebra of the invariants under $W$ is spanned by $n$ algebraically independent homogeneous elements whose degrees $d_{i}$ are uniquely determined by $R$.

The reader who would like more details about root systems may refer to Bourbaki [4].

B] Macdonald's conjectures. We are now able to state the simplest of the Macdonald's conjectures. As in the $A_{n-1}$ case we will denote by $e^{\alpha}$ the element of $Z(\Lambda)$ corresponding to $\alpha$, for each element $\alpha$ of $\Lambda$, the lattice associated to $R$.

Conjecture. If $R$ is a reduced root system, then

$$
C T \prod_{\alpha \in R}\left(1-e^{\alpha}\right)^{k}=\prod_{i=1}^{n}\left(\begin{array}{c}
k d_{i} \\
k
\end{array}\right)
$$

for any non-negative integer $k$.

There are $n$ parameters in the Dyson conjecture and only one in this one. So Macdonald [17] proposed also a several parameters conjecture for any root system (unfortunately not equivalent to the Dyson conjecture when $R=A_{n-1}$ ).

Conjecture. Let $R$ be a (non-necessarily reduced) root system and, for each $\alpha \in R$, let $k_{|\alpha|}$ be a non-negative integer depending only on $\alpha$ 's norm. Putting $\rho_{k}=1 / 2 \sum_{\alpha \in R^{+}} k_{|\alpha|} \alpha$ and $k_{|\alpha / 2|}=0$ if $\alpha / 2 \notin R$, we have:

(BIG MAC)

$$
C T \prod_{\alpha \in R}\left(1-e^{\alpha}\right)^{k_{|\alpha|}}=\prod_{\alpha \in R} \frac{\left(\alpha^{v}\left(\rho_{k}\right)+k_{|\alpha|}+1 / 2 k_{|\alpha / 2|}\right) !}{\left(\alpha^{v}\left(\rho_{k}\right)+1 / 2 k_{|\alpha / 2|}\right) !}
$$

In the same way there are $q$-Macdonald's conjectures. 


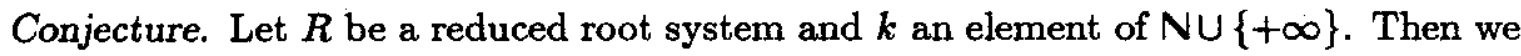
have:

(qMAC)

$$
C T \prod_{\alpha \in R^{+}}\left(q e^{\alpha}\right)_{k}\left(e^{-\alpha}\right)_{k}=\prod_{i}\left[\begin{array}{c}
k d i \\
k
\end{array}\right]
$$

where $\left[\begin{array}{l}n \\ k\end{array}\right]$ denotes the $q$-binomial coefficient $(q)_{n} /(q)_{k}(q)_{n-k}$.

There is also a $q$-version of the (BIG MAC) conjectures but it requires some more tools.

C. Affine root systems and Morris's conjectures. Let $E$ be a finite dimensional affine euclidean space, with translation space $V$ and let $F$ be the vector space of the affine forms on $E$. So each element $f$ of $F$ may be defined on $E$ by $f(a+v)=f(a)+\langle D f, v\rangle$, for any scalar $a$ and any vector $v$, and denoting by $\langle$,$\rangle the scalar product on V$; the vector of $V$ denoted by $D f$ is called the gradient of $f$. Then one can define on $F$ a semi-definite positive symmetric bilinear form by $\langle f, g\rangle=\langle D f, D g\rangle$. For $f$ non-constant, the real number $\langle f, f\rangle$ is non-zero and so the linear form $f^{\vee}$ (defined as above by $f^{\vee}(g)=2\langle f, g\rangle /\langle f, f\rangle$ ) and the isometry $w_{f}$ (defined by $\left.w_{f}(g)=g-f^{\vee}(g) f\right)$ are well-defined.

An affine root system is a subset $S$ of $F$ satisfying the four following axioms:

(ARS 1) $S$ spans $F$ and does not contain any constant;

(ARS 2) If $f \in S$, then $w_{f}(S)=S$;

(ARS 3) If $f, g \in S$, then $f^{\vee}(g) \in \mathbf{Z}$;

(ARS 4) The group $W(S)$ acts properly on $E$.

As above $W(S)$ denotes the Weyl group of $S$, spanned by the $w_{f}$ and it acts on $E$ via $w_{f}(x)=x-2 f(x) /\langle f, f\rangle D f$. One can notice that each axiom (ARS i) is the exact affine analogue of the axiom (RS $\mathrm{i}$ ), for $i=1,2,3,4$.

An affine root system will be said to be reduced if and only if two proportional roots are equal or opposite, and irreducible if it cannot be written as the disjoint union of two non-empty orthogonal subsets.

Let $S$ be an affine root system, with gradient root system $\sum$ (i.e. $\sum=\{D f: f \in S\}$ ). A basis $B$ of $S$ will be a basis of $F$ such that the coordinates of any element of $S$ are either all non-negative or all non-positive. The existence of such a basis is insured by the theory of infinite dimensional Lie algebras. For a given basis $B$ we define a chamber $C$ by $C=\{x \in E: b(x)>0, \forall b \in B\}$. For each $f$ in $S$, let us define $u_{f}$ to be the smallest real number such that $f+a_{f} \in S$. This number $u_{f}$ depends only on $D f$ and so we can talk about $u_{\alpha}$, for $\alpha$ in $\sum$. Let us put again:

$$
\begin{aligned}
\Sigma_{0} & =\{\alpha \in \Sigma: 2 \alpha \in \Sigma\}, \\
\bar{\Sigma} & =\{\alpha \in \Sigma: 2 \alpha \notin \Sigma\}, \\
\bar{\Sigma}^{+} & =\bar{\Sigma} \cap \Sigma^{+},
\end{aligned}
$$


where $\sum^{+}$is the set of the positive roots of $\sum$, relatively to $C$. Let us also denote by $\chi_{0}$ the characteristic function of $\sum_{0}$.

A labelling of $S$ will be a function $k: S \rightarrow \mathrm{N}$ such that $k(f)=k(g)$ whenever $D f=D g$ or $\langle f, f\rangle=\langle g, g\rangle$. As $k$ depends only on the gradients, we can also define it on $\sum$; on $\sum_{0}$ the function $k$ is constant, say $k_{0}$. Then let us put $\rho_{k}=\frac{1}{2} \sum_{\alpha \in \Sigma^{+}}+k(\alpha) \alpha$ and

$$
S_{k}=\left\{f \in S: 0<f(x)<u_{f} k(f), \forall x \in C\right\}
$$

we are now able to state the most general conjecture.

Conjecture. If $S$ is irreducible and reduced, then we have:

\section{(MORRIS)}

$$
\begin{aligned}
& C T \prod_{f \in S_{k}}\left(1-e^{f}\right) \\
& \quad=\frac{1}{(g ; g)_{k_{0}}^{l}} \prod_{\alpha \in \sum^{+}} \frac{\left(q^{u_{\alpha}} ; q^{u_{\alpha}}\right)_{\alpha^{v}\left(\rho_{k}\right)+k(\alpha)+x_{0}(\alpha) k_{0}}\left(q^{u_{\alpha}} ; q^{u_{\alpha}}\right)_{\alpha^{v}}\left(\rho_{k}\right)-k(\alpha)+k_{0}}{\left(q^{u_{\alpha}} ; q^{u_{\alpha}}\right)_{\alpha^{v}\left(\rho_{k}\right)}\left(q^{u_{\alpha}} ; q^{u_{\alpha}}\right)_{\alpha^{v}}\left(\rho_{k}\right)+k_{0}}
\end{aligned}
$$

The reader who would like to get more results about affine root systems or more explanations about this last conjecture should refer to Morris's thesis [18].

D. Examples. For $\sum=A_{n-1}$, Morris's conjecture reduces to the equal parameters version of the Dyson conjecture. However, related to this root system, he stated another conjecture, for which he was able to prove the case $q=1$ :

(MORRIS)

$$
\begin{aligned}
& C T \prod_{i=1}^{n}\left(\frac{x_{0}}{x_{i}}\right)_{a}\left(q \frac{x_{i}}{x_{0}}\right)_{b} \prod_{1 \leq i<j \leq n}\left(\frac{x_{i}}{x_{j}}\right)_{c}\left(q \frac{x_{j}}{x_{i}}\right)_{c} \\
& =\prod_{j=0}^{n-1} \frac{(q)_{a+b+j c}(q)_{(j+1) c}}{(q)_{a+j c}(q)_{b+j c}(q)_{c}}
\end{aligned}
$$

This conjecture is an "intermediate conjecture" between $A_{n-1}$ And $A_{n}$ is the sense that the case $a=b=0$ gives the (q-MAC) conjecture for $A_{n-1}$ and the case $a=b=c$ gives the (q-MAC) conjecture for $A_{n}$.

In order to illustrate the differences between the several forms of the Macdonald's conjectures, let us study the root system $G_{2}$, the only root system of dimension 2 (so graphically representable) for which the solutions are non-trivial. 


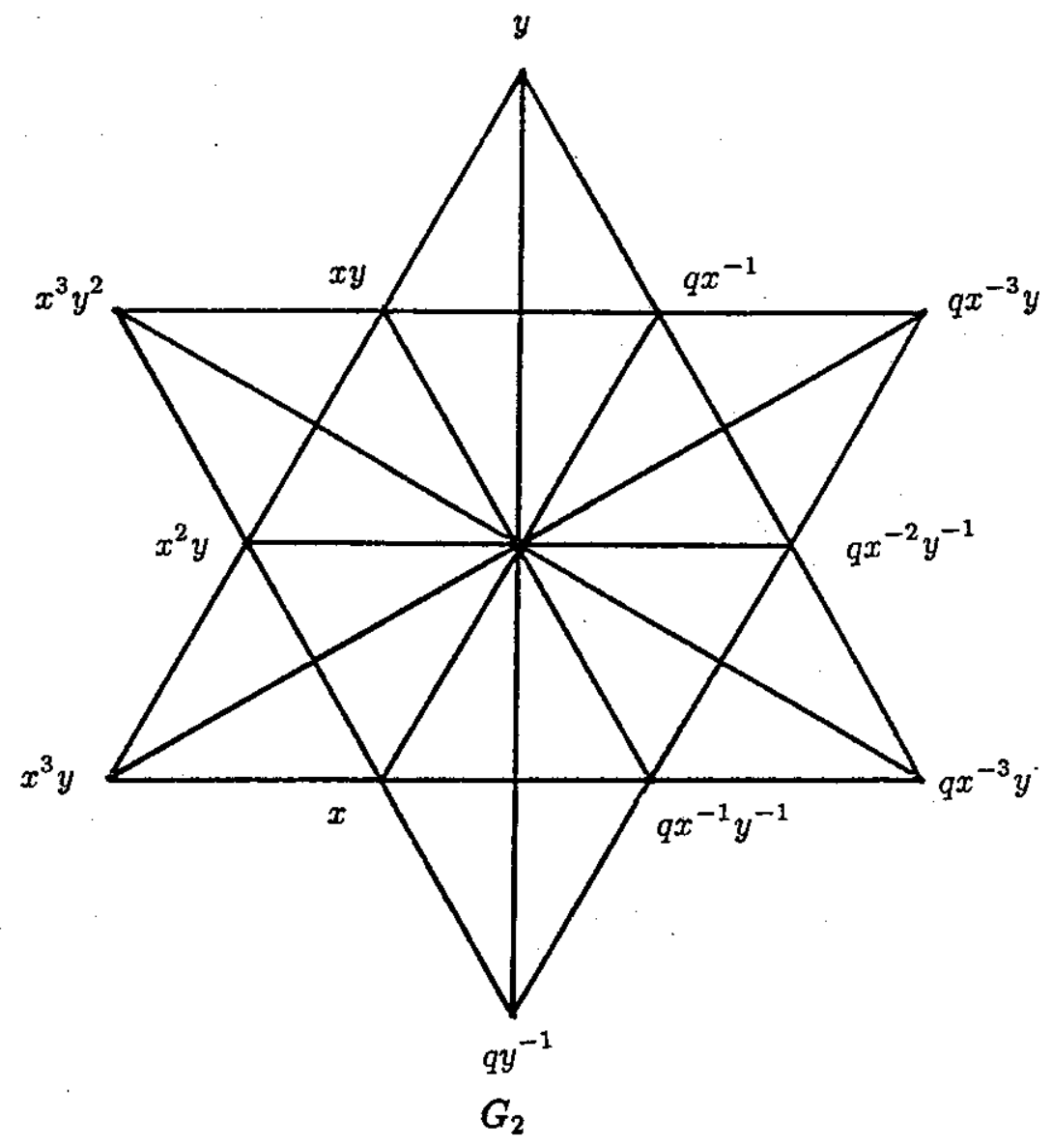

The conjectures related to $G_{2}$ have the following forms:

(BIG MAC)

$$
\begin{aligned}
C T & (1-x)^{a}\left(1-x^{-1}\right)^{a}\left(1-x^{2} y\right)^{a}\left(1-x^{-2} y^{-1}\right)^{a}(1-x y)^{a}\left(1-x^{-1} y^{-1}\right)^{a} \\
& (1-y)^{b}\left(1-y^{-1}\right)^{b}\left(1-x^{3} y\right)^{b}\left(1-x^{-3} y^{-1}\right)^{b}\left(1-x^{3} y^{2}\right)^{b}\left(1-x^{-3} y^{-2}\right)^{b} \\
& =\frac{(3 a+3 b) !(3 b) !(2 a) !(2 b) !}{(2 a+3 b) !(a+2 b) !(a+b) ! a ! b !^{2}}
\end{aligned}
$$

(q BIG MAC)

$$
\begin{aligned}
& C T(x ; q)_{a}\left(q x^{-1} ; q\right)_{a}\left(x^{2} y ; q\right)_{a}\left(q x^{-2} y^{-1} ; q\right)_{a}(x y ; q)_{a}\left(q x^{-1} y^{-1} ; q\right)_{a} \\
& \quad(y ; q)_{b}\left(q y^{-1} ; q\right)_{b}\left(y^{3} y ; q\right)_{b}\left(q x^{-3} y^{-1} ; q\right)_{b}\left(x^{3} y^{2} ; q\right)_{b}\left(q x^{-3} y^{-2} ; q\right)_{b} \\
& \quad=\frac{(q ; q)_{3 a+3 b}(q ; q)_{2 a}(q ; q)_{2 b}(q ; q)_{3 b}}{(q ; q)_{2 a+3 b}(q ; q)_{a+2 b}(q ; q)_{a+b}(q ; q)_{a}(q ; q)_{b}^{2}}
\end{aligned}
$$

(MORRIS)

$$
\begin{aligned}
& C T(x ; q)_{a}\left(q x^{-1} ; q\right)_{a}\left(x^{2} y ; q\right)_{a}\left(q x^{-2} y^{-1} ; q\right)_{a}(x y ; q)_{a}\left(q x^{-1} y^{-1} ; q\right)_{a} \\
& \quad\left(y ; q^{3}\right)_{b}\left(q^{3} y^{-1} ; q^{3}\right)_{b}\left(x^{3} y ; q^{3}\right)_{b}\left(q^{3} x^{-3} y^{-1} ; q^{3}\right)_{b}\left(x^{3} y^{2} ; q^{3}\right)_{b}\left(q^{3} x^{3} y^{2} ; q^{3}\right)_{b} \\
& \quad=\frac{(q ; q)_{3 a+3 b}(q ; q)_{2 a}(q ; q)_{3 b}\left(q^{3} ; q^{3}\right)_{a+3 b}\left(q^{3} ; q^{3}\right)_{2 b}\left(q^{3} ; q^{3}\right)_{a}}{q ; q)_{2 a+3 b}(q ; q)_{a+3 b}(q ; q)_{a}^{2}\left(q^{3} ; q^{3}\right)_{a+2 b}\left(q^{3} ; q^{3}\right)_{a+b}\left(q^{3} ; q^{3}\right)_{b}^{2}}
\end{aligned}
$$




\section{E. Results:}

General results: In February 1988, Tom Koornwinder claimed that Erik Opdam from the University of Utrecht just proved the (BIG MAC) conjecture, by using the shift operators developed in his $\mathrm{Ph} . \mathrm{D}$. thesis. Unfortunately none of the specialists we talked with about it has received a copies of this paper.

In his original paper [17] Macdonald proved the cases $k=1,2$ or $\infty$ of the ( $\mathrm{q}-\mathrm{MAC}$ ) conjecture. Since september 1987 a rumor spread that Feigin, a Russian mathematician, was able to prove it for all $k$ but again no paper reached us.

In 1983 Phil Hanlon [13], considering the Macdonald's conjectures for the infinite families of root systems, proved they were "asymptotically" true.

The root system $A_{n}$ : Considering Macdonald's conjectures in this case as part of Dyson conjecture allows us to collect all the results from the first part at this point. However Stembridge (May 1986, [20]) gave a direct proof of the (q-MAC) conjecture, which is not connected to the Dyson conjecture.

The ( $\mathrm{q}-\mathrm{MAC}$ ) conjecture may also be viewed as a special case of the (MORRIS) conjecture given in the previous section. Morris (1982, [18]) was able to prove his conjecture when $q=1$ and in January 1986 Habsieger [11] and Kadell [15] gave independently a proof of the $q$-case. Later Zeilberger (May 1987, [25]) extended Stembridge's proof to Morris's conjecture.

The infinite families $B_{n}, C_{n}, D_{n}$ : They are considered as special cases of a larger (non-reduced) root system called $B C_{n}$. Macdonald $(1982$, [17]) was able to prove the (BIG MAC) conjecture for the root system $B C_{n}$ and Kadell $(1986,[16])$ gave the proof of the $q$-case.

The exceptional root systems: There are not any specific results for the root systems $E_{6}, E_{7}$ and $E_{8}$; only the "general results" apply. In August 1987 Garvan [8] gave a direct computer-proof of the (BIG MAC) conjecture for $F_{4}$. The root system $G_{2}$ was the most successful: Habsieger (1986, [12]) and Zeilberger (1986, [23]) gave independently and simultaneously a proof of the ( $q$-BIG MAC) conjecture, later followed by Garvan (1987, [7]). A proof of the (MORRIS) conjecture related to $G_{2}$ was also discovered by Zeilberger (1987, [24]).

The natural question that occurs after this enumeration of results is: what is remaining? Taking for granted the two claims given among the general results, the only open cases remaining are the ( $q$-BIG MAC) and the (MORRIS) conjectures for the exceptional root system $F_{4}$.

\section{THIRD PART: Selberg's integral.}

A. The ordinary case. In an almost forgotten paper [19] Selberg evaluated the 
following integral:

$$
\begin{aligned}
& \int_{0}^{1} \cdots \int_{0}^{1} \prod_{i=1}^{n} t_{i}^{x-1}\left(1-t_{i}\right)^{y-1} \prod_{1 \leq<j \leq n}\left|t_{i}-t_{j}\right|^{2 z} d t_{1} \ldots d t_{n} \\
& =\prod_{j=0}^{n-1} \frac{\Gamma(x+j z) \Gamma(y+j z) \Gamma((j+1) z+1)}{\Gamma(x+y+(n+j-1) z) \Gamma(z+1)}
\end{aligned}
$$

Recently Aomoto (1983, [2]) gave another proof of this result.

Macdonald [17] used this evaluation to prove the $B C_{n}$ case of his conjecture as follows: replace each basis vector by $e^{2 i \pi \theta_{j}}$ and integrate on $[0,1]^{n}$ for the Lebesgue measure $d \theta_{1} \ldots d \theta_{n}$; in this integral do the change of variables $t_{j}=\sin ^{2} \pi \theta_{j}$ to get Selberg's integral.

Selberg's integral may be also used to prove Morris's identity when $q=1$ by using the following trick.

We have $\int_{0}^{1} t^{\lambda-1} d t=1 / \lambda$ for $\operatorname{Re} \lambda>0$. Then, by analytic continuation, you can define for $\lambda \in C \backslash\{0\}$ the quantity $\int_{0}^{1} t^{\lambda-1} d t$ and you have for every integer $n$

$$
\lim _{\epsilon \rightarrow 0} \epsilon \int_{0}^{1} t^{\epsilon-1} t^{n} d l t=\left\{\begin{array}{lll}
1 & \text { if } & n=0 \\
0 & \text { if } & n \neq 0
\end{array}\right.
$$

Thus CT $f=\lim _{\epsilon \rightarrow 0} \epsilon \int_{0}^{1} f(t) t^{\epsilon-1} d t$ and the connection between Selberg's integral and Morris's identity is established.

The $q$-analogue.

$$
\begin{array}{ll}
\text { Let us define } & \Gamma_{q}(a)=\frac{(q)_{\infty}}{\left(q^{\epsilon}\right)_{\infty}}(1-q)^{1-a}, \quad \text { for } a \in C \backslash Z . \\
\text { and } \int_{0}^{1} f(t) d_{q} t=(1-q) \sum_{n=0}^{\infty} f\left(q^{n}\right) q^{n}
\end{array}
$$

Askey (1980, [3]) proposed the following $q$-extension of Selberg's integral:

$$
\begin{gathered}
\int_{0}^{1} \cdots \int_{0}^{1} \prod_{i=1}^{n} t_{i}^{x-1} \frac{\left(t_{i} q\right)_{\infty}}{\left(t_{i} q^{y}\right)_{\infty}} \prod_{1 \leq i<j \leq n} t_{i}^{2 k}\left(\frac{t_{j}}{t_{i}} q^{1-k}\right)_{2 k} d_{q} t_{1} \ldots d_{q} t_{n} \\
=q^{k x\left(\begin{array}{c}
n \\
2
\end{array}\right)+2 k^{2}\left(\begin{array}{c}
n \\
8
\end{array}\right)} \prod_{j=0}^{n-1} \frac{\Gamma_{q}(x+j k) \Gamma_{q}(y+j k) \Gamma_{q}((j+1) k+1)}{\Gamma_{q}(x+y+(n+j-1) k) \Gamma_{q}(k+1)}
\end{gathered}
$$

As usual we get the ordinary case by letting $q$ tend to 1 . This conjecture was proved in 1986 by Habsieger [11], by adapting Selberg's proof, and Kadell [15], by adapting Aomoto's proof. Both of them were able to deduce Morris's identity from this $q$-integeral. Indeed the same trick works, for $\int_{0}^{1}\left(t^{\lambda-1} d q t=(1-q) /\left(1-q^{\lambda}\right)\right.$ and thus we have CT $f=\lim _{\epsilon \rightarrow 0}$ $\left(1-q^{\epsilon}\right) /(1-q) \int_{0}^{1} t^{\epsilon-1} f(t) d_{q} t$. Another application of this integeral is the $G_{2}$-case of the Macdonald's conjectures. 


\section{REFERENCES}

[1] GEORge E. ANDREws, Problems and prospects for basic hypergeometric functions, in The Theory and Applications of Special Functions, edited by Richard Askey, Academic Press, New York, 1975, pp. 191-224.

[2] KazUнiко Аомото, Jacobi polynomials associated with Selberg integrals, SIAM J. Math. Anal., t 18 (1987), pp. 545-549.

[3] RICHARD ASKEY, Some basic hypergeometric extensions of integrals of Selberg and Andrews, SIAM J. Math. Anal, t 11 (1980), pp. 938-951.

[4] Nicolas Bourbaki, Groupes et algèbres de Lie, Chapitres IV, V, VI, Hermann, Paris, 1968

[5] David M. Bressoud and Ian Goulden, Constant term identities extending the $q$-Dyson theorem, Trans. Amer. Math. Soc., t 291 (1985), pp. 203-228.

[6] Freeman Dyson, Statistical theory of the energy levels of complex systems I, J. Math. Phys, t. 3 (1962), pp. 140-156.

[7] Frank Garvan, A Beta Integral associated with the Root System $G_{2}$, IMA Preprint Series, \# 360.

[8] Frank Garvan, A proof of the Macdonald-Morris root system conjecture for $F_{4}$, IMA Preprint Series, \# 370.

[9] I.J. Good, Short proof of a conjecture of Dyson, J. Math. Phys., t. 11 (1970), p. 1886.

[10] J. Gunson, Proof of a conjecture of Dyson in the statistical theory of energy levels, J. Math. Phys, t.3 (1962), pp. 752-753.

[11] Laurent Habsieger, Une q-intégrale de Selberg-Askey, SIAM J. Math. Anal., to appear.

[12] LaURent Habsteger, La $q$-conjecture de Macdonald-Morris pour $G_{2}$, C.R. Acad. Sc. Paris, t.303, Série 1,6 (1986), pp. 211-214.

[13] Pall Hanlon, The proof of a limiting case of Macdonald's root system conjecture, Proc. Lond. Math. Soc., t 49 (1984), pp. 170-182.

[14] Kevin KADELL, Andrews $q$-Dyson conjecture: $n=4$, Trans. Amer. Math. Soc, t. 290 (1985), pp. 127-144.

[15] KeVIN KADELL, A proof of Askey's conjectured q-analog of Selberg's integral and a conjecture of Mortis, SIAM J. Math. Anal, 19 (1988).

[16] KEVIN KaDELL, A proof of the $q$-Macdonald-Morris conjecture for $B C_{n}$, preprint.

[17] Ian MAcDonald, Some conjectures for root systems, SIAM J. Math. Anal, t. 13 (1982), pp. 988-1007.

[18] WALTER MoRRIs, Constant term identities for finite or affine root systems, conjectures and theorems, Ph.D. thesis, University of Wisconsin, Madison, Wisconsin.

[19] AtLe Selberg, Bemerkninger on et multpelt integral, Norsk. Mat. Tidsskr, t. 26 (1944), pp. 71-78.

[20] JoHN STEMBRIDGE, A short proof of Macdonald's conjecture for the root systems of type $A$, preprint.

[21] Kenneth G. Wilson, Proof of a conjecture of Dyson, J. Math. Phys.; t.3 (1962), pp. 1040-1043.

[22] Doron Zellberger, A combinatorial proof of Dyson's conjecture, Discrete Math., t. 41 (1982), pp. 317-321.

[23] Doron ZeILBERGer, A proof of the $G_{2}$ case of Macdonald's root system-Dyson conjecture, SIAM J. Math. Anal..

[24] Doron Zeilberger, A unified approach to Macdonald's root system conjectures, preprint.

[25] Doron ZetLberger, A Stembridge-Stanton style elementary proof of the Habsieger-Kadell-q-Morris formula, preprint.

[26] Doron Zellberger and David M. Bressoud, A proof of Andrews $q$-Dyson conjecture, Discrete Math., t. 54 (1985), pp. 201-224. 